\title{
Modified Suturing Techniques in Carotid Endarterectomy for Reducing the Cerebral Ischemic Time
}

\author{
Sung-Pil Joo, ${ }^{*}$ Yong-Hwan Cho, ${ }^{*}{ }^{+}$Yong-Jun Lee, You-Sub Kim, Tae-Sun Kim \\ Department of Neurosurgery, Chonnam National University Hospital, Chonnam National University Medical School, Gwangju, Korea
}

Objective : Carotid endarterectomy (CEA) is an effective surgical procedure for treating symptomatic or asymptomatic patients with carotid stenosis. Many neurosurgeons use a shunt to reduce perioperative ischemic complications. However, the use of shunting is still controversial, and the shunt procedure can cause several complications. In our institution, we used two types of modified arteriotomy suture techniques instead of using a shunt.

Methods : In technique 1, to prevent ischemic complications, we sutured a third of the arteriotomy site from both ends after removing the plaque. Afterward, the unsutured middle third was isolated from the arterial lumen by placing a curved Satinsky clamp. And then, we opened all the clamped carotid arteries before finishing the suture. In technique 2, we sutured the arteriotomy site at the common carotid artery (CCA). We then placed a curved Satinsky clamp crossing from the sutured site to the carotid bifurcation, isolating the unsutured site at the internal carotid artery (ICA). After placing the Satinsky clamp, the CCA and external carotid artery (ECA) were opened to allow blood flow from CCA to ECA. By opening the ECA, ECA collateral flow via ECA-ICA anastomoses could help to reduce cerebral ischemia.

Results : The modified suture methods can reduce the cerebral ischemia directly (technique 1) or via using collaterals (technique 2). The modified arteriotomy suture techniques are simple, safe, and applicable to almost all cases of CEA.

Conclusion : Two modified arteriotomy suture techniques could reduce perioperative ischemic complications by reducing the cerebral ischemic time.

Key Words : Carotid stenosis · Endarterectomy, Carotid · Suture techniques · Collateral circulation.

\section{INTRODUCTION}

Carotid endarterectomy (CEA) is an effective surgical procedure for preventing ischemic stroke in selected patients with symptomatic or asymptomatic carotid stenosis ${ }^{2,16,17)}$. But CEA has the risk of perioperative ischemic complications due to deliberate artery occlusion during clamping of the internal carotid artery (ICA) and thrombosis due to distal low flow ${ }^{12,15)}$.

- Received : March 1, 2020 • Accepted : March 30, 2020

- Address for reprints : Tae-Sun Kim

Department of Neurosurgery, Chonnam National University Hospital, 42 Jebong-ro, Dong-gu, Gwangju 61469, Korea

Tel : +82-62-220-6608, Fax : +82-62-224-9865, E-mail : taesun1963@yahoo.co.kr, ORCID : https://orcid.org/0000-0001-7134-073X

*These authors contributed equally to this work.

${ }^{\dagger}$ Current affiliation : Department of Neurosurgery, Cerebrovascular Center, Dong-A University Hospital, Dong-A University College of Medicine, Busan, Korea 


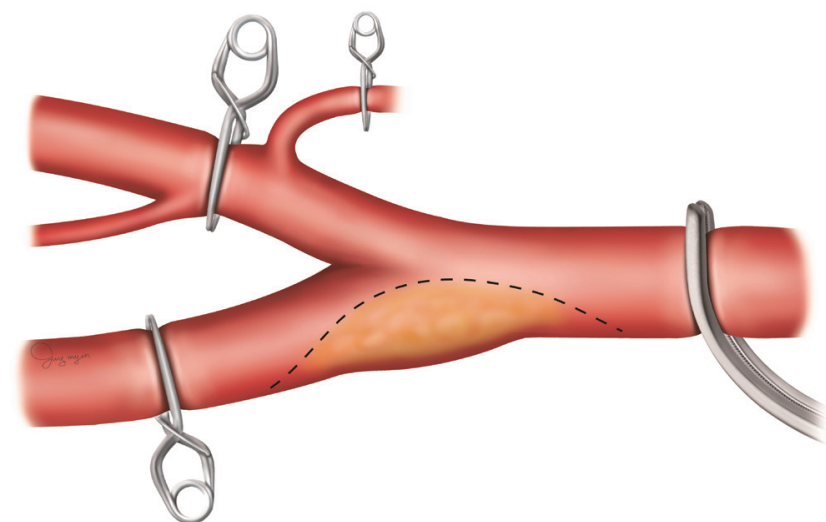

Fig. 1. In conventional carotid endarterectomy, the ICA, CCA, and ECA are clamped in that order. From incision on the carotid artery to closure of the arteriotomy, the entire process of endarterectomy is performed in the condition that the ICA, CCA, and ECA are clamped. After finishing the complete closure of the arteriotomy site, the ICA, ECA, and CCA are unclamped. ICA : internal carotid artery, CCA : common carotid artery, ECA : external carotid artery.
To maintain cerebral blood flow during carotid clamping, some neurosurgeons routinely use a shunt, while others use a shunt selectively based on transcranial Doppler, electro encephalography, carotid stump back pressure, or neurological changes under local anesthesia ${ }^{1)}$. However, the shunt procedure also leads to complications such as distal embolic events during shunt placement, arterial dissection, acute occlusion, and difficult exposure of the distal end of a high plaque ${ }^{1,4,14)}$. Overall, the use of shunt is still controversial, and some neurosurgeons do not use a shunt at all ${ }^{9}$. At our institution, we used two modified closure methods of incision on the carotid artery instead of using a shunt to reduce the cerebral ischemic period during CEA. In conventional CEA, the ICA, the common carotid artery (CCA) and the external carotid artery (ECA) are clamped in that order. And then the entire process of endarterectomy is performed in the condition that the ICA,
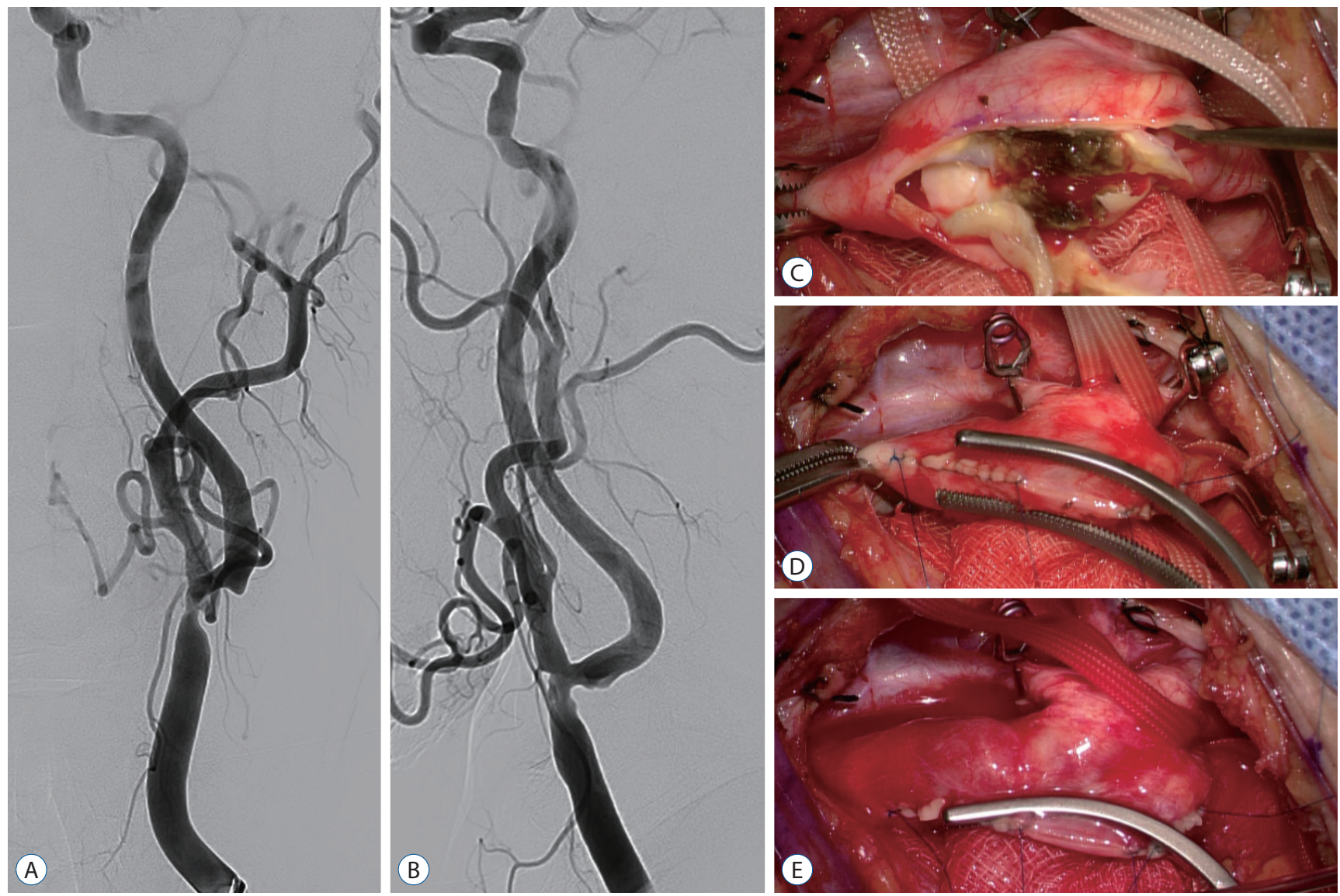

Fig. 2. A and B : Cerebral angiography revealed NASCET 55\% stenosis in the left distal CCA including the ICA origin site. The lesion was accompanied by luminal irregularity ( $\mathrm{A}$ : anteroposterior view, $\mathrm{B}$ : lateral view). $\mathrm{C}$ : The atheroma was an unstable plaque with ulceration. D : Applying a Satinsky clamp to isolated unsutured site from the carotid artery lumen. $E$ : After applying a Satinsky clamp, the ECA, ICA, and CCA were opened before finishing the closure of the arteriotomy site. NASCET : The North American Symptomatic Carotid Endarterectomy Trial, CCA : common carotid artery, ICA : internal carotid artery, ECA : external carotid artery. 
CCA and ECA are clamped. After complete closure of the endarterectomy site, the clamps are removed in the reverse order of clamping (Fig. 1). At our institution, we are exploring ways to reduce the carotid artery clamping time by removing the clamp of the ICA or ECA before finishing the complete suture of the arteriotomy site. In this paper, we introduce two methods with cases.

\section{MATERIALS AND METHODS}

This study design was approved by the Institutional Review Board of Chonnam National University Hospital (CNUH2020-038) and fulfilled all of the requirements for patient anonymity. We retrospectively reviewed total 55 cases who underwent modified suture techniques during CEA at our institution between June 2018 and November 2019. We used two modified closure methods of incision on the carotid artery and each method is described below with a case.

\section{Surgical technique 1 with a case}

A 79-year-old man presented with recurrent syncope. He had a history of hypertension and diabetes mellitus. His neurologic examination revealed no deficit. There was no sign of acute cerebral infarction on the magnetic resonance imaging (MRI). However, the magnetic resonance angiography (MRA) demonstrated severe stenosis of the left CCA and ICA. Cerebral angiography revealed 55\% segmental moderate stenosis (The North American Symptomatic Carotid Endarterectomy Trial, NASCET) in the left distal CCA, including the left ICA origin site (Fig. 2A and B). The plaque appeared unstable because of accompanying luminal irregularity, which led to our
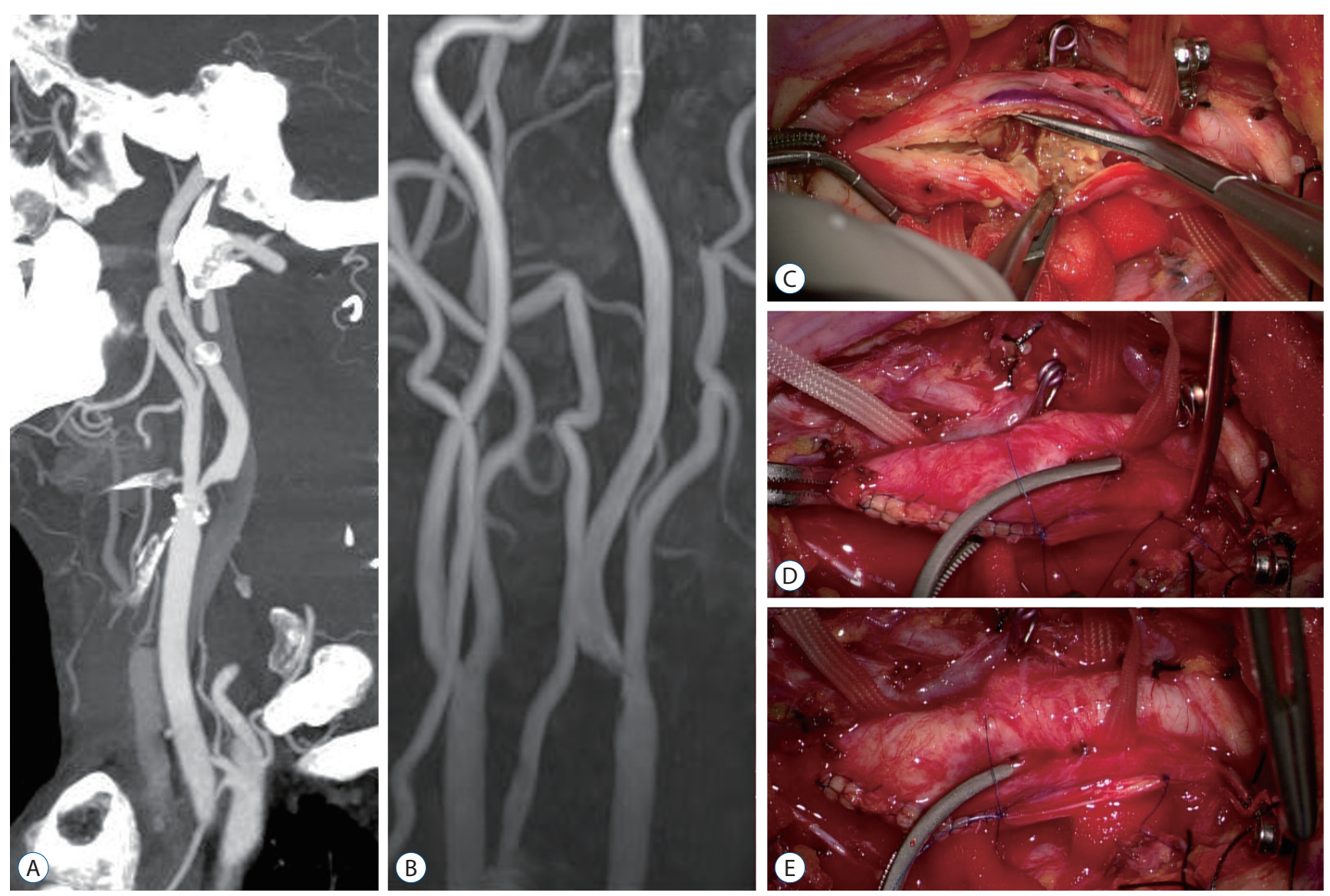

Fig. 3. A : Computed tomography angiography revealed severe stenosis of the left proximal cervical ICA (NASCET 80\%) with calcification. B : Magnetic resonance angiography also demonstrated severe proximal ICA stenosis with ulceration. $C$ : Intraoperative finding of unstable atheroma with ulceration. D : Applying a Satinsky clamp to isolate the unsutured site at the ICA from the CCA lumen. E : After applying a Satinsky clamp, the ECA and CCA were opened before finishing the complete suture of the arteriotomy site. ICA : internal carotid artery, NASCET : The North American Symptomatic Carotid Endarterectomy Trial, CCA : common carotid artery, ECA : external carotid artery. 
decision to perform a CEA.

Under general anesthesia, the patient was placed in the supine position. Intraoperative monitoring devices were used to check the motor evoked potential (MEP) and regional oxygen saturation $\left(\mathrm{rSO}_{2}\right)$. The patient's head was placed on a gel doughnut, and slightly extended and turned 15 degrees to the right. A curvilinear incision was made along the anterior border of the sternocleidomastoid muscle. And then the dissection was performed toward the carotid artery. After identification of the ICA, CCA, and ECA, the clamps were placed. The ICA and ECA were closed with Sugita clips, and CCA was occluded with a Satinsky clamp. The arteriotomy was performed on the atheroma and extended to ICA and CCA side, until normal lumens were identified at both sides. Intraoperative findings of the atheroma indicated an unstable plaque. The atheroma had a necrotic core (Fig. 2C), and careful endarterectomy was performed. ICA stump back pressure after ECA and CCA clamping was low at 12/11 $\mathrm{mmHg}$. The baseline $\mathrm{rSO}_{2}$ was $57 \%$ before ICA clamping and decreased to $46 \%$ immediately after the ICA clamping. During endarterectomy, systolic blood pressure increased to over $150 \mathrm{mmHg}$; however the $\mathrm{rSO}_{2}$ was still remained near the high $40 \%$. To prevent ischemic complications, we had to open the ICA as soon as possible as a shunt was not used. After removing the residual debris and irrigating the endarterectomy surface, the primary closure was started. A continuous locking suture was begun at the distal end of the arteriotomy and advanced to distal a third. Another suture was started at the proximal end of the arteriotomy and ran to the proximal a third in the same manner. The remaining middle third was not sutured. The ICA was opened briefly to allow backflow and to wash out debris. The same procedure was performed for the ECA and then for the CCA. Afterward, the unsutured middle third was isolated from the arterial lumen by placing curved a Satinsky clamp (Fig. 2D). The clamps on the ECA, CCA, and ICA were removed in this order (Fig. 2E). By isolating the unsutured arteriotomy site with the Satinsky clamp, we were able to open the ICA before finishing the primary closure, and the $\mathrm{rSO}_{2}$ immediately recovered to $64 \%$. The surgery was completed uneventfully. During the entire CEA, the MEP was maintained in the normal range, and there was no complication after the surgery.

\section{Surgical technique 2 with a case}

A 74-year-old man with intermittent headache and dizziness underwent a brain MRI, MRA, and neck computed tomography angiography (CTA). The MRA and CTA revealed severe stenosis of the proximal cervical ICA (NASCET $80 \%$ ) with calcification and ulceration (Fig. 3A and B). The stenosis was severe, and ulceration was present. Therefore, we decided to perform a CEA.

Under general anesthesia, preoperative preparation was carried out in the same way as in the above-described case. Intraoperative monitoring devices for MEP and $\mathrm{rSO}_{2}$ were used, and the operation was performed on the patient's left neck. After identification of the ICA, ECA, and CCA, clamps were placed on the ECA and CCA. The ICA stump back pressure was $60 / 37 \mathrm{mmHg}$. After clamping of ICA, a conventional endarterectomy was performed, which revealed that the plaque was unstable (Fig. 3C). After removing the atheroma and the residual debris and irrigating of endarterectomy surface, primary closure was initiated. In this case, a continuous locking suture was started at the proximal end of the arteriotomy and advanced distally. When the suture reached around the carotid bifurcation, the ICA, ECA and CCA were opened for a few seconds to wash out debris. Then, the ICA and the unsutured site were isolated from the CCA and ECA by placing a curved Satinsky clamp crossing from the sutured site to the carotid bifurcation (Fig. 3D). The next step was opening the CCA and ECA (Fig. 3E). As the ECA has multiple anastomoses with the ICA and its branches, the ischemic risk could be reduced by opening the CCA and ECA early. Following this, the suturing was continued. The $\mathrm{rSO}_{2}$ was $60 \%$ just prior to the ICA clamping, decreased to $56 \%$ after ICA clamping, maintained around 55\% during the procedure, and increased to $60 \%$ after opening the CCA and ECA. The ICA stump back pressure measured before the last suture also increased to $82 / 48$ $\mathrm{mmHg}$. The surgery was completed uneventfully without any postoperative complications. The MEP was maintained within the normal range during the entire procedure.

\section{RESULTS}

In our institution, technique 1 was applied to 10 patients and technique 2 was applied to 45 patients from June 2018 to November 2019. There was only one case of perioperative 


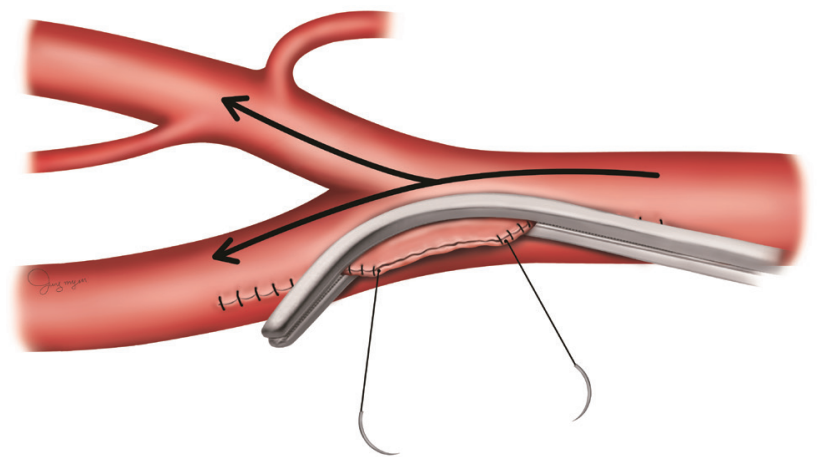

Fig. 4. Suture technique 1. The key point of this method is opening the clamps early at the CCA, ICA, and ECA before finishing the complete closure of the arteriotomy, by placing a curved Satinsky clamp at the unsutured site. Black arrows indicate direct blood flow from CCA to ICA. To enable a smooth blood flow, the Satinsky clamp should be placed at the CCA. This method is more applicable in cases that the arteriotomy extends to the CCA. CCA : common carotid artery, ICA : internal carotid artery, ECA : external carotid artery.

complication. The patient was 80 -year-old man and underwent CEA using technique 2 on right carotid artery without a shunt. After the surgery, he presented left hemiplegia. However, there was no acute infarction or hemorrhage in brain MRI and CTA. We assumed that the symptom was caused by hyper-perfusion. After rehabilitation, the patient recovered to motor grade IV.

\section{DISCUSSION}

CEA is an effective surgery for primary or secondary prevention of ischemic stroke in selected patients with symptomatic or asymptomatic carotid stenosis. The benefits of CEA have been demonstrated in major trials ${ }^{2,16,17)}$. The benefit of the CEA procedure relies on the low perioperative stroke rates $^{6,8,16)}$. According to Riles et al. ${ }^{10)}$, among 3062 primary carotid endarterectomies, perioperative stroke occurred in 66 cases (2.2\%). Ten of these cases were related to ischemia during carotid artery clamping ${ }^{10)}$. Carotid occlusion during clamping in CEA could cause cerebral hypoperfusion or intraoperative thrombosis; both mechanisms are related to perioperative strokes ${ }^{15}$. As the efficacy of CEA is determined by the minimization of perioperative stroke rate ${ }^{5}$, it is important for surgeons to reduce the cerebral ischemic time during CEA. Many neurosurgeons use a shunt to prevent cerebral ischemic complications. However, by placing a shunt, potential prob-

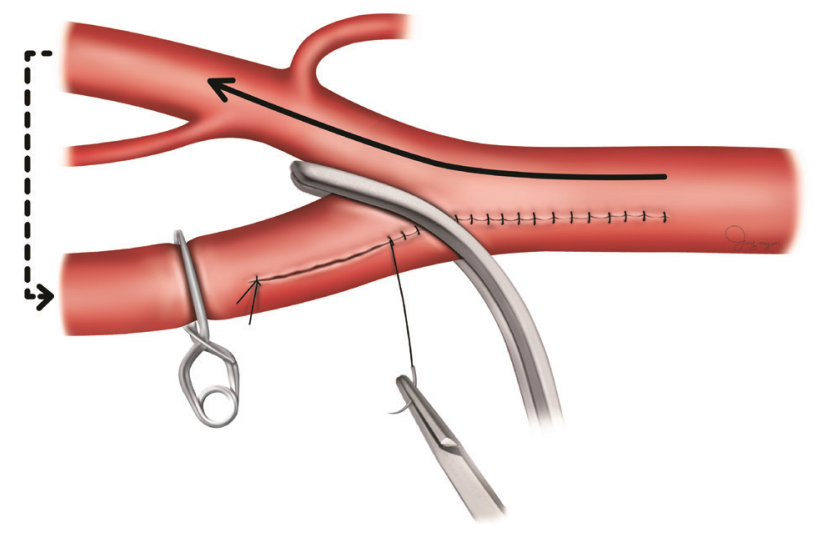

Fig. 5. Suture technique 2. This method is using collateral blood flow via multiple anastomoses between the ICA and ECA. First, the arteriotomy at the CCA is sutured. After that, the unsutured site at the ICA is isolated using a curved Satinsky clamp. Afterward, the CCA and ECA are opened to enable blood flow from the CCA to the ECA. Black arrow indicates blood flow from CCA to ECA and dotted black arrow means collateral flow via ECA-ICA anastomoses. This method is easy to apply in cases where the plaque is located at the ICA side and the arteriotomy is not extended to the CCA. ICA : internal carotid artery, ECA : external carotid artery, CCA : common carotid artery.

lems, such as dissection of the arterial wall, difficulty of securing a shunt, or dislodgement of the shunt, may arise ${ }^{10)}$. The use of shunting may also cause air embolism at shunt introduction $^{15)}$. Therefore, the use of shunting is still controversial. Some neurosurgeons do not use a shunt at all and have reported good results without a shunt ${ }^{3,9,11,15)}$. In our institution, we do not use a shunt, but aim to reduce the carotid clamping time to prevent cerebral ischemia. To achieve this, we have used two types of modified suture techniques for arteriotomy to reduce the cerebral ischemic time.

Technique 1 is the early opening of ICA using a Satinsky clamp before finishing the suture of the arteriotomy. The method is as follows : 1) after removing the plaque, the suture starts at the distal end of the arteriotomy and advances to the distal third of arteriotomy with a continuous locking suture. 2) Another suture starts at the proximal end of the arteriotomy and proceeds to proximal third with a continuous locking suture. 3) Open the ICA clamp temporarily to wash out debris and then sequentially open the ECA, CCA clamp for a short time. 4) Isolate the unsutured middle third with a curved Satinsky clamp from the carotid artery lumen. 5) Open the ECA, CCA, and ICA clamp in that order. And 6) finish the suture at the middle third and then open the Satinsky clamp. To enable a smooth blood flow from the CCA to the ICA, the curved 
Satinsky clamp should be applied at the CCA. If the Satinsky clamp is applied at the ICA, the ICA would collapse because of its small lumen. Technique 1 is applicable to cases where the atheroma plaque is extended to the CCA site (Fig. 4). In case 1, ICA clamping time was 22 minutes and 55 seconds. It took an additional four minutes and 53 seconds from the ICA unclamping to the removal of the curved Satinsky clamp for finishing the remnant suture. We could reduce the cerebral ischemic time by approximately 5 minutes before finishing the arteriotomy suture, which is a reduction of $18.7 \%$. Futhermore, the $\mathrm{rSO}_{2}$ was also improved. After ICA clamping, $\mathrm{rSO}_{2}$ dropped from $57 \%$ to $46 \%$ and immediately rose to $64 \%$ after applying the curved Satinsky clamp and unclamping of the ICA.

Technique 2 uses the anastomoses between the ICA and the ECA. The ipsilateral ECA has multiple anastomoses with the ipsilateral ICA, which can be an important collateral in cases of ipsilateral ICA occlusion ${ }^{7,13)}$. In technique 2, by opening the ECA clamp before finishing the arteriotomy suture, blood flow via ECA-ICA anastomoses could reduce brain ischemia. The method works as follows : 1) after removing the plaque, the suture starts at the proximal end of the arteriotomy and advances distally to around the CCA bifurcation with a continuous locking suture. 2) Open the ICA clamp temporarily to wash out debris and then sequentially open the ECA and CCA clamp for a short time. 3) Isolate the unsutured site at the ICA with a curved Satinsky clamp from the CCA and ECA. 4) Open the ECA and CCA clamp and allow blood flow to the ECA. And 5) finish the suture at the ICA and then open the ICA clamp and the Satinsky clamp. Technique 2 is applicable to cases when the arteriotomy is not extended far to the CCA because the plaque is located mainly at the ICA. The shorter the incision on the CCA, the faster the Satinsky clamp can be applied. In case 2, the total clamping time was 26 minutes with six minutes from the unclamping the ECA and CCA to finishing suture and unclamping the ICA. We could supply blood flow via the ECA collaterals 6 minutes before finishing the complete closure of the arteriotomy site. The $\mathrm{rSO}_{2}$ decreased from $60 \%$ to $56 \%$ immediately after the ICA clamping, but rose back to $60 \%$ after ECA unclamping. ICA stump back pressure improved from 60/37 mmHg after ICA clamping to $82 / 48 \mathrm{mmHg}$ after ECA unclamping. In case 2, in our opinion, the collateral flow via ECA and the increased systolic blood pressure from $120 \mathrm{mmHg}$ to more than $160 \mathrm{mmHg}$ af- ter ICA clamping could have affected the result of equal or more improved $\mathrm{rSO}_{2}$ and ICA stump back pressure after opening the ECA only than the $\mathrm{rSO}_{2}$ and ICA stump back pressure before ICA clamping.

It is unclear whether the modified suture method alone can improve the functional outcome after the CEA because many other factors can affect the results, such as blood pressure and the flow from contralateral ICA. However, we think that the modified suture methods can reduce the cerebral ischemia directly (technique 1 ) or via using collaterals (technique 2 ), and it might reduce perioperative complications due to the ICA clamp. Finally, the modified arteriotomy suture techniques are simple, and do not need other devices such as a shunt. Thus, the modified arteriotomy suture techniques do not cause severe complications. These methods are applicable to almost all cases of CEA.

\section{CONCLUSION}

CEA is the golden standard treatment for symptomatic and asymptomatic patients with severe carotid stenosis. The efficacy of CEA is determined by the minimization of perioperative complications. The modified arteriotomy suture techniques are simple and easy and could help reduce the perioperative stroke rate by reducing the cerebral ischemic time.

\section{CONFLICTS OF INTEREST}

No potential conflict of interest relevant to this article was reported.

\section{INFORMED CONSENT}

This type of study does not require informed consent.

\section{AUTHOR CONTRIBUTIONS}

\author{
Conceptualization : TSK \\ Data curation : YJL \\ Formal analysis : YSK
}


Funding acquisition : SPJ

Methodology: TSK

Project administration : SPJ

Visualization : YHC

Writing - original draft : YHC

Writing - review \& editing : SPJ

\section{ORCID}

Sung-Pil Joo

Yong-Hwan Cho

Yong-Jun Lee

You-Sub Kim

Tae-Sun Kim https://orcid.org/0000-0002-4983-0174

https://orcid.org/0000-0002-0840-0039

https://orcid.org/0000-0002-5086-4494

https://orcid.org/0000-0001-5410-7418

https://orcid.org/0000-0001-7134-073X

\section{References}

1. Aburahma AF, Mousa AY, Stone PA : Shunting during carotid endarterectomy. J Vasc Surg 54 : 1502-1510, 2011

2. Barnett HJ, Taylor DW, Eliasziw M, Fox AJ, Ferguson GG, Haynes RB, et al. : Benefit of carotid endarterectomy in patients with symptomatic moderate or severe stenosis. North American Symptomatic Carotid Endarterectomy Trial Collaborators. N Engl J Med 339 : 1415-1425, 1998

3. Boontje $\mathrm{AH}$ : Carotid endarterectomy without a temporary indwelling shunt: results and analysis of back pressure measurements. Cardiovasc Surg 2 : 549-554, 1994

4. Hans SS, Catanescu I : Selective shunting for carotid endarterectomy in patients with recent stroke. J Vasc Surg 61 : 915-919, 2015

5. Jacobowitz GR, Rockman CB, Lamparello PJ, Adelman MA, Schanzer A, Woo $D$, et al. : Causes of perioperative stroke after carotid endarterectomy: special considerations in symptomatic patients. Ann Vasc Surg 15 : 19-24, 2001

6. Lawrence PF, Alves JC, Jicha D, Bhirangi K, Dobrin PB : Incidence, tim- ing, and causes of cerebral ischemia during carotid endarterectomy with regional anesthesia. J Vasc Surg 27 : 329-334; discussion 335-337, 1998

7. Liebeskind DS : Collateral circulation. Stroke 34 : 2279-2284, 2003

8. North American Symptomatic Carotid Endarterectomy Trial Collaborators, Barnett HJM, Taylor DW, Haynes RB, Sackett DL, Peerless SJ, et al. : Beneficial effect of carotid endarterectomy in symptomatic patients with high-grade carotid stenosis. N Engl J Med 325 : 445-453, 1991

9. Rerkasem K, Rothwell PM : Routine or selective carotid artery shunting for carotid endarterectomy (and different methods of monitoring in selective shunting). Cochrane Database Syst Rev (4) : CD000190, 2009

10. Riles TS, Imparato AM, Jacobowitz GR, Lamparello PJ, Giangola G, Adelman MA, et al. : The cause of perioperative stroke after carotid endarterectomy. J Vasc Surg 19 : 206-214; discussion 215-216, 1994

11. Samson RH, Showalter DP, Yunis JP : Routine carotid endarterectomy without a shunt, even in the presence of a contralateral occlusion. Cardiovasc Surg 6 : 475-484, 1998

12. Thompson JE : Complications of carotid endarterectomy and their prevention. World J Surg 3 : 155-165, 1979

13. van Laar PJ, van der Grond J, Bremmer JP, Klijn CJ, Hendrikse J : Assessment of the contribution of the external carotid artery to brain perfusion in patients with internal carotid artery occlusion. Stroke 39 : 30033008, 2008

14. Woodworth GF, McGirt MJ, Than KD, Huang J, Perler BA, Tamargo RJ : Selective versus routine intraoperative shunting during carotid endarterectomy: a multivariate outcome analysis. Neurosurgery 61 : 1170-1176; discussion 1176-1177, 2007

15. Wu TY, Anderson NE, Barber PA : Neurological complications of carotid revascularisation. J Neurol Neurosurg Psychiatry 83 : 543-550, 2012

16. Young B, Moore WS, Robertson JT, Toole JF, Ernst CB, Cohen SN, et al. : An analysis of perioperative surgical mortality and morbidity in the asymptomatic carotid atherosclerosis study. ACAS Investigators. Asymptomatic Carotid Atherosclerosis Study. Stroke 27 : 2216-2224, 1996

17. Randomised trial of endarterectomy for recently symptomatic carotid stenosis: final results of the MRC European Carotid Surgery Trial (ECST). Lancet 351 : 1379-1387, 1998 